\title{
Pensar Diferente
}

\section{Luis Augusto Pisco ${ }^{1}$ \\ André Ribeiro ${ }^{2}$ \\ Eliedna Barbosa ${ }^{3}$}

É com muita satisfação que apresentamos o número especial da Revista do Gestão \& Saúde de 2020 que tem como tema "Reflexões sobre a Pandemia COVID-19 e seus Impactos na Administração Pública e na Gestão dos Serviços Públicos de Saúde" em virtude das consequências causadas pelo novo coronavírus de forma global. Os pesquisadores e sistemas de serviços de saúde de todo o mundo têm enfrentado e buscado estratégias e ações práticas para lidar com a transmissão e óbitos causados pelo surto desta pandemia. O Núcleo de Estudos em Educação e Promoção à Saúde da Universidade de Brasília, através de sua revista, oferece este fascículo aos esforços de todos os pesquisadores que incansavelmente vêm buscando soluções inovadoras para combater a COVID-19. Este fascículo começa com uma reflexão "Pensar diferente" que discute as reformas da Atenção Primária de Saúde (APS) no contexto de Portugal e Brasil.

Nos últimos anos, muitos Países, entre os quais Portugal e o Brasil procuraram fazer reformas da sua Atenção Primária de Saúde (APS) visando melhorar o acesso, a eficiência e de uma forma geral a qualidade dos cuidados. Investiu-se em infraestruturas, equipamentos e recursos humanos procurando obter proximidade e qualidade para a população e maior motivação e melhores condições de trabalho e de remuneração (pagamento ligado ao desempenho) para os profissionais de saúde.

Em Portugal, esta reforma iniciou-se em 2005, e em 2006 iniciou-se a criação de uma rede de Unidades de Saúde Familiares. Teve como objetivo o aumento da acessibilidade aos cuidados

\footnotetext{
${ }^{1}$ Faculdade de Ciências Médicas, Nova Medical School. Lisboa Portugal. Presidente da Administração Regional de Saúde de Lisboa e Vale do Tejo (ARSLVT), Lisboa. ORCID http://orcid.org/0000-0002-9007-8949 - Email: luispisco@ mail.telepac.pt

2 Educador Físico e Pedagogo, Mestre e Doutor em Ciências da Saúde Pesquisador do Ceam/Nesprom UnB - Brasília (Brasil), ORCID https://orcid.org/0000-0002-2167-9345 - Email: andreribeiro@unb.br

${ }^{3}$ Doutora e mestra em Ciências Contábeis pela Universidade de Brasília (UnB), pesquisadora do Núcleo de Estudos em Gestão de Serviços/UnB, Professora e pesquisadora do Grupo de Estudos em Avaliação em Tecnologias em Saúde (ATS) do Núcleo de Tecnologias Estratégicas em Saúde (NUTES) da Universidade Estadual da Paraíba (UEPB) ORCID https://orcid.org/0000-0001-6334-6584 - Email: eliedna.barbosa@gmail.com
} 
de saúde, melhorar a continuidade dos cuidados, aumentar a motivação dos Médicos de Família e dos profissionais de saúde em geral, estimulando também os cuidados domiciliários e criando uma Rede Nacional de Cuidados Continuados Integrados.

A situação excecional e os desafios inesperados criados pela pandemia SARS-CoV-2 e pela infeção por COVID-19 veio alterar significativamente a situação epidemiológica e do sistema de saúde no seu todo (relegando para segundo plano as reformas que vinham a ser desenvolvidas na APS), quer por força da própria doença, que implicou uma resposta específica e imediata dos serviços de saúde, quer por força da suspensão da atividade programada, com o propósito de prevenir a transmissão do vírus e combater a potencial calamidade pública resultante da atividade pandémica. Apesar de num segundo momento (maio de 2020) ter sido determinado o reagendamento de toda a atividade assistencial programada não realizada por força da pandemia COVID-19 esse objetivo foi só parcialmente conseguido.

Este novo enquadramento está a testar a capacidade do Serviço Nacional de Saúde (SNS) e dos profissionais de APS para absorver, responder com eficiência e adaptar-se a choques e mudanças estruturais, ao mesmo tempo que desempenham as novas atividades que lhes estão cometidas. Esta capacidade, tem sido demonstrada a dois níveis distintos: resposta em continuidade e capacidade de resiliência.

A resposta em continuidade da APS foi muito condicionada pela suspensão da atividade assistencial programada e, resumidamente, pode dizer-se que entre janeiro e setembro de 2020 toda a atividade da APS diminuiu 22\%, em relação ao período homólogo de 2019 (consultas presenciais de médicos, enfermeiros e outros técnicos de saúde, consultas no domicílio e consultas de situações agudas).

De momento os profissionais de APS estão perante o imperativo de garantir cuidados de saúde a todos os usuários, sobretudo os que viram as suas consultas e tratamentos diferidos no tempo por força da pandemia, a reconfiguração do atendimento nos serviços para evitar/reduzir a transmissão da COVID-19 e o desempenho de novas funções e solicitações nomeadamente o aumento exponencial de contatos à distância. 
As teleconsultas tornaram-se na forma preferencial de contacto entre profissionais de saúde e doentes. Em Lisboa e Vale do Tejo (LVT) até setembro de 2020 foram feitas 3.284.535 teleconsultas, que representam mais 1.590 .823 que em 2019 (período homólogo). Foram também entregues 1.848 VPN para que os profissionais possam trabalhar a partir de casa evitando diversos riscos. A pandemia acelerou o que teriam sido anos (ou mesmo algumas décadas) de mudança, tendo sido provavelmente os nove meses mais transformacionais que o nosso sistema de saúde já enfrentou.

A questão da resiliência dos sistemas de saúde e dos seus profissionais não é nova. Para além disso neste momento está a ser influenciada por duas condicionantes que estiveram anteriormente ausentes: a) os confinamentos (nacionais ou locais) e as novas formas de organização do trabalho (teletrabalho e equipas-espelho, por exemplo); b) a repercussão económica e social das medidas adotadas para "aplanar a curva epidemiológica".

Nesta capacidade de resiliência e de adaptação quase diária, dos profissionais de APS, deve-se reafirmar as novas atividades assumidas pela APS em tempos de pandemia e que são muito exigentes. Na Região de Saúde de LVT que abrange 3.6 milhões de pessoas (cerca de um terço da população Portuguesa) 95\% dos doentes com COVID 19 confirmado são tratados em casa e cerca de 5\% são internados num Hospital e 0,7\% são internados em cuidados intensivos. Daqui se pode perceber a importância do bom desempenho da Atenção Primária nesta pandemia.

Outra área importante são os testes e as campanhas de testagem na comunidade. À data de 14 de setembro Portugal tinha uma taxa de 222.527 testes por milhão de habitantes (sexta maior entre países da união Europeia). O envolvimento dos profissionais de APS tem sido essencial sobretudo no contexto dos Estabelecimentos Residenciais para Pessoas Idosas.

Como muito recentemente escreveu o Conselho Nacional de Saúde "Nenhum país, sistema de saúde ou instituição estava preparado para uma pandemia com a dimensão e com a repercussão que se observa. Sobretudo, a pandemia continua a constituir, por um período que se estima longo, uma dura prova para todos os países e setores, a nível global."

Entre a continuidade e a resiliência, certamente que se identificam ações e inovações organizacionais que não devem terminar com a crise pandémica e devem ter um papel mais 
importante no futuro. Pela capacidade de resiliência demonstrada os profissionais de saúde revelaram capacidade para lidar com o choque sistémico e para adotarem novas formas de trabalho, numa transformação rápida e sem precedentes. Olhar para frente e encontrar novas soluções implica pensar diferente.

Na seção de Artigos originais, Planejamento na incerteza: Variáveis para estimar custos médicos direitos de paciente suspeito ou confirmado para COVID-19, de Patrícia Silva da Silva, Carine Garcia Daniel, Roger dos Santos Rosa e Ronaldo Bordin descrevem os protocolos de manejo clínico de pacientes suspeitos e confirmados para COVID-19 no Estado do Rio Grande do Sul, no período de 30 de janeiro a 20 de maio de 2020, e identificam variáveis para o cálculo dos custos médicos diretos no atendimento desses pacientes.

Em seguida, Comunicação em saúde dos planos privados durante a pandemia, de Flavia Harumi Ramos Tanaka e Wilson Marques Vieira Junior objetivou verificar, por meio de um questionário, a percepção dos usuários com relação à comunicação em saúde pelas operadoras durante a pandemia.

Na seção Artigo de pesquisa, Uma análise estatística comparativa das evidências de subnotificação da COVID-19 no Brasil, de Igor Ferreira do Nascimento, Alex Rodrigues do Nascimento e Peng Yaohao desenvolveram um modelo estatístico que incorpora aspectos temporais e espaciais, o qual analisou a dispersão da COVID-19 a partir de um epicentro para três unidades federativas brasileiras e quatro estados norte-americanos; a influência relativa da malha rodoviária para a dispersão da COVID-19 nesses estados também foi verificada.

Na seção Ensaio teórico, A gestão de suprimentos na administração pública diante a pandemia do novo coronavírus, de Thiago Fidelis de Sousa Furriel, Cristiane Teixeira Sendim e Adriano Bernardo de Sá Roriz realizaram uma pesquisa documental sobre as aquisições públicas a partir de dois modelos, centralizado e descentralizado, e os possíveis impactos no enfrentamento à pandemia. 
Na sequência, Produção pública de medicamentos no Brasil no contexto da pandemia da COVID-19, de Paula Teixeira Pinto Ferreira Neto e Camila Rocha da Cunha discutiram a produção pública de medicamentos no âmbito dos laboratórios oficiais e seus desafios no contexto da pandemia de COVID-19, utilizando como base os medicamentos do estudo Solidarity.

Já o artigo, Serviços farmacêuticos nos países incluídos no Health In Transition (HIT): uma análise comparada, de Ingrid Britto Sousa, Vinícius Nunes Carvalho, Hebert Luan Pereira Campos dos Santos e Nília Maria de Brito Lima Prado analisa a conformação dos serviços farmacêuticos em quatro países da União Europeia - Espanha, França, Portugal e Itália -, a organização das políticas e os processos de regulamentação do acesso aos medicamentos com base nas publicações do HIT.

Na seção Artigo teórico empírico, A pejotização em saúde na macrorregião norte do Paraná e suas implicações com a COVID-19, de João Felipe Marques da Silva, Brígida Gimenez Carvalho e Stela Maris Lopes Santini analisam a pejotização de recursos humanos na área da saúde na macrorregião norte do Paraná e apontam possíveis implicações provocadas pela pandemia de COVID-19, devido à maior fragilização e precarização das relações de trabalho dos profissionais vinculados aos serviços de saúde por essa modalidade.

Por sua vez, As fragilidades do federalismo cooperativo na crise do COVID-19, de João Mendes da Rocha Neto discuti, a partir das narrativas de governantes, impactos sobre a coordenação da política de saúde no período em que o país enfrenta a pandemia do Covid-19, tendo como pano de fundo as relações federativas.

Impactos da pandemia da COVID-19 na dispensação de medicamentos pela assistência farmacêutica da regional de Pirapora, de Giovana Gonçalves Pereira, Patrícia de Oliveira, André Soares Santos e Keli Bahia Felicíssimo Zocratto descrevem as medidas de flexibilização e adequação adotadas pela Coordenação da Assistência Farmacêutica (CAF) da Gerência Regional de Saúde de Pirapora, durante a pandemia causada pela Covid-19, com base nas legislações do Ministério da Saúde, Secretaria de Estado da Saúde de Minas Gerais e da Agência Nacional de Vigilância Sanitária (Anvisa). 
Por fim, As múltiplas faces da pandemia: reflexos sobre o coronavírus na saúde pública do Brasil, de Rafael Zaneripe de Souza Nunes, Marieli Mezari Vitali, Cristiane Damiani Tomasi e Lisiane Tuon buscam apontar as principais características do vírus e seus reflexos, principalmente no Brasil.

Os textos aqui, além de contribuírem por meio de diferentes perspectivas sobre o combate a pandemia do novo coronavírus, permitiram uma integração de pesquisadores de diversas áreas do conhecimento.

O desejo dos editores deste fascículo é que o exercício de produção do conhecimento aqui apresentado possa estimular e incentivar tantos outros pesquisadores que estão observando ou vivenciando este ímpar momento na história da humanidade.

Boa leitura. 